\title{
Control of Particles in Microelectrode Devices
}

\author{
Idan Tuval, ${ }^{1}$ Igor Mezić, ${ }^{2}$ Frédéric Bottausci, ${ }^{2}$ Yanting T. Zhang, ${ }^{2}$ Noel C. MacDonald, ${ }^{2}$ and Oreste Piro ${ }^{1}$ \\ ${ }^{1}$ Institut Mediterrani d'Estudis Avançats, CSIC-UIB, E-07071 Palma de Mallorca, Spain \\ ${ }^{2}$ Department of Mechanical \& Environmental Engineering, University of California, Santa Barbara, California 93106-5070, USA
}

(Received 13 October 2004; revised manuscript received 4 August 2005; published 1 December 2005)

The impact of the convective fluid motion induced by the electric fields on the dielectrophoretic manipulation of particles is investigated theoretically and experimentally. By means of a simplified model a channel with a periodic array of microelectrodes we show that electroconvective flows induce the formation of traps for particles, providing a dynamical mechanism to control microparticles in such devices. We demonstrate experimentally the theoretically predicted dynamical phenomena.

Dielectric particles suspended in a dielectric media are polarized under the action of electric fields. If the field is spatially inhomogeneous, it exerts a net force on the polarized particle known as dielectrophoretic (DEP) [1]. This force depends upon the temporal frequency and spatial configuration of the field as well as on the dielectric properties of both the medium and the particles. Dielectrophoresis is an increasingly popular efficient method to separate particles in microflows [2]. DEP forces can be switched on and off to selectively capture cells, bacteria, spores, DNA, proteins, and other matter. One could envision, for instance, an application using DEP to capture a suspected pathogen which then is shuttled to a selected area of the microfluidic device where its DNA is extracted and analyzed.

Since the dielectrophoretic mobility of a particle scales directly with its surface area, the manipulation of smaller particles requires larger gradients of the electric fields. Nevertheless, by using microfabricated electrodes to generate large electric field gradients, it is now possible to move submicron particles by means of DEP [3,4]. However, large electric field gradients may strongly interact with the background media creating, by several electrohydrodynamic effects, flows whose drag perturbs the particle trajectories. An understanding of this disturbance is thus crucial to predict and control it in developing applications of DEP to specific microfluidic devices. On the other hand, the combined dynamics induced by both advection and electric forces is a largely unexplored but interesting field of research.

In this Letter we first introduce a model where the DEP particle dynamics produced by such arrays is perturbed by the advection due to a realistic model of the corresponding electrohydrodynamic convective flow. By means of this model we then predict an important dynamical consequence of the perturbing flow: the appearance of trapping zones from where particles cannot escape.

We consider a simple but commonly used configuration of electrodes array for which a closed-form solution of the electric field and the DEP force was derived in [5]. It consists of a periodic array of long parallel microelectrodes as depicted in Fig. 1(a). The time-averaged DEP force is

$$
\begin{aligned}
\left\langle F_{\mathrm{DEP}}\right\rangle & =2 \pi a^{3} \epsilon_{m} \operatorname{Re}[K(\omega)] \nabla|E|^{2} \\
\nabla|E|^{2} & =\frac{\pi^{3} V_{0}^{2}}{K^{2}(\cos (\pi / 4)) d^{3}} \operatorname{Re}\left[\begin{array}{c}
i z k(\bar{z}) k^{\prime}(z) \\
-z k(\bar{z}) k^{\prime}(z)
\end{array}\right] \\
k(z) & =\left(\frac{z}{1-2 z \cos (\pi / 2)+z^{2}}\right)^{1 / 2} \\
z & =\exp (\pi(i x-y) / d),
\end{aligned}
$$

where $E$ is the rms electric field, $a$ is the particle radius, $\omega$ is the angular field frequency, and $\operatorname{Re}[z]$ indicates the real part of the complex number $z$. The factor $K(\omega)$ is a measure of the effective polarizability of the particle, known as the Clausius-Mossotti factor, given by $K(\omega)=$ $\left(\epsilon_{p}^{*}-\epsilon_{m}^{*}\right) /\left(\epsilon_{p}^{*}+2 \epsilon_{m}^{*}\right)$ where $\epsilon_{p}^{*}$ and $\epsilon_{m}^{*}$ are the complex permittivities of the particle and the medium, respectively. The complex permittivity is defined as $\epsilon^{*}=\epsilon-i(\sigma / \omega)$, where $i=\sqrt{-1}, \epsilon$ is the permittivity, and $\sigma$ is the conductivity of the dielectric.

The Clausius-Mossotti factor depends on the dielectric properties of the particle and the medium, and on the frequency of the applied field. Variations in this factor

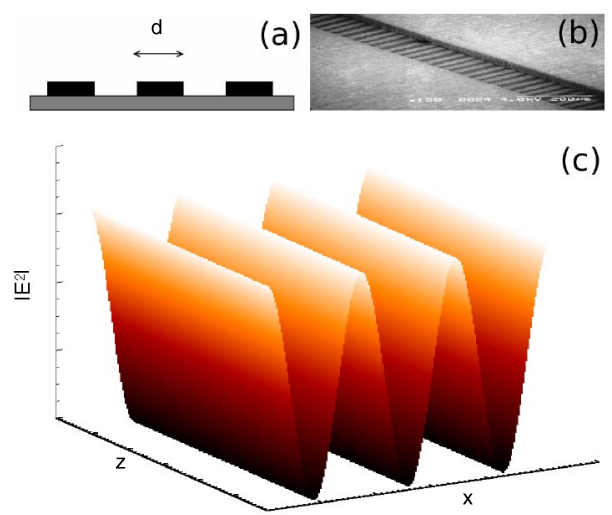

FIG. 1 (color online). (a) The arrangement of an interdigitated electrode array. (b) SEM image of the titanium DEP chip with 24 parallel electrodes. (c) Electric field strength, $|E|^{2}$, in a plane $10 \mu \mathrm{m}$ above the electrodes. 
give rise to a DEP force that is frequency dependent and unique to each particle type. For a sphere, the real part of $K(\omega)$ is bounded by the limits $-\frac{1}{2}<\operatorname{Re}[K(\omega)]<1$. When $\operatorname{Re}[K(\omega)]>0$ the induced force points toward the high electric field at the electrode surfaces and is known as positive-DEP ( $p$-DEP). Conversely, when $\operatorname{Re}[K(\omega)]<0$ (negative- or $n$-DEP), the force points in the direction of decreasing field strength and the particles are repelled from the electrodes edge [Fig. 2(a)].

In our configuration the electric field has local minima above the center of the electrodes whereas it reaches the strongest values at their edges [Fig. 1(c)]. In the absence of fluid flow, the particles experiencing $p$-DEP collect at the strong field points at the electrode edges. On the other hand, particles pushed away from the electrodes by $n$-DEP reach an equilibrium position away from the electrodes where the vertical component of the DEP force is balanced by buoyancy. Since the horizontal component decays much faster than the vertical one, in dynamical terms these equilibrium positions form, in practice, a continuous line of fixed points.

However, electric fields induce fluid motions through several electrohydrodynamic effects. The most important of those that occur in microelectrode devices are electrothermal convection and ac electro-osmosis. The former appears due to a nonuniform Joule heating of the fluid which leads to gradients of its permittivity and conductivity. The applied electric fields acting on the permittivity and conductivity gradients generate electrical body forces that induce the flow [6]. The latter, instead, is caused by electrical stresses in the diffuse double layer of charges accumulated above the electrodes [7]. Whether electrothermal or ac-electro-osmotic flows dominate the motion of fluid in the device depends mainly on the frequency of the applied electric field, ac electro-osmosis being dominant at a frequency range several orders of magnitude below the charge relaxation frequency $\left(\omega_{c} \equiv \sigma / \epsilon\right)$. In any of these situations, the electrohydrodynamic forces dominate the buoyancy forces at typical microfluidic system sizes $(d<$ $300 \mu \mathrm{m}$ ) [8]. For a careful choice of the applied frequency, the induced fluid flows will have a minimal effect but in the DEP manipulation and/or separation of submicron parti-

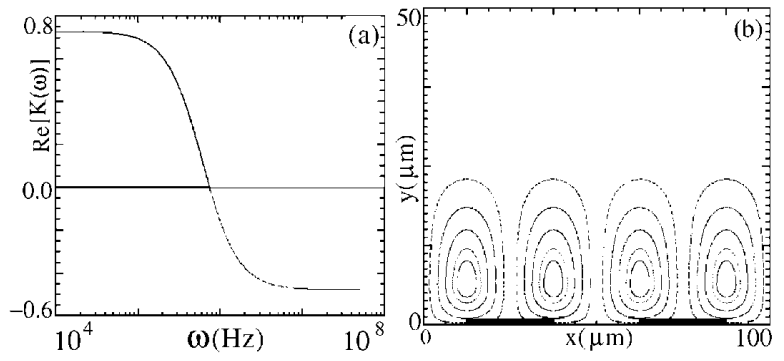

FIG. 2. (a) Plot of the real part of the Clausius-Mossotti function for $\epsilon_{m}=80 \epsilon_{0}, \sigma_{m}=0.001 \mathrm{~S} \mathrm{~m}^{-1}, \epsilon_{p}=2.5 \epsilon_{0}$, and $\sigma_{p}=0.009 \mathrm{~S} \mathrm{~m}^{-1}$. (b) Streamlines of the cellular flow used in the model. cles one is usually forced to use frequencies for which the fluid flow generated electrohydrodynamically has to be taken into account. We will show that this is not necessarily an annoyance, since the induced dynamical properties can be creatively used as a mechanism to control microparticles.

Experiments and numerical simulations of the coupled electrothermohydrodynamic problem in devices with interdigitated array of electrodes [4,7,9-11] show that both electrothermal and ac-electro-osmotic flows consist of convective rolls centered at the electrode edges and provide good estimates for their strength and frequency dependence. Near the electrodes, the fluid velocity $\boldsymbol{u}_{0}$ ranges from 1 to $100 \mu \mathrm{m} \mathrm{s}^{-1}$ decaying exponentially with the transversal distance to the electrodes. Additionally, the flow satisfies no-slip boundary condition at the bottom of the device $\left(\boldsymbol{u}_{x}=\boldsymbol{u}_{y}=0\right)$ and both the horizontal component of the velocity and the normal derivative of the vertical one vanish at the symmetry planes $\left(\boldsymbol{u}_{x}=\partial \boldsymbol{u}_{y} /\right.$ $\partial n=0)$. In order to analyze the impact on the DEP dynamics of the observed cellular flow we mimic this with a simple model fulfilling all the above mentioned conditions. This flow, depicted in Fig. 2(b), comes from the stream function:

$$
\psi_{\text {steady }}=\boldsymbol{u}_{0} y^{2} e^{-y / \beta} \cos (\pi x)
$$

which ensures its incompressibility, $\nabla \boldsymbol{u}=0$. The parameter $\beta$ controls the vertical position of the center of the rolls.

In a device of a characteristic length $d \simeq 20 \mu \mathrm{m}$, with flow velocities $\boldsymbol{u}_{0} \simeq 10 \mu \mathrm{m} \mathrm{s}^{-1}$, fluid viscosity $\nu \simeq$ $10^{-6} \mathrm{~m}^{2} \mathrm{~s}^{-1}\left(\eta=\rho \nu \simeq 10^{-3} \mathrm{~kg} \mathrm{~m}^{-1} \mathrm{~s}^{-1}\right)$, and micrometer particles $a \simeq 1 \mu \mathrm{m}$, the particle's Stokes number is of order $\mathrm{St}=\left(2 a^{2} u / 9 \nu d\right) \simeq 10^{-6}$, which implies that inertial effects can be neglected. For particles of a few hundreds of $\mathrm{nm}$, Brownian motion can also be neglected when compared to DEP forces [6]. Therefore, the velocity of the particles is determined by only the DEP, buoyancy and drag forces:

$$
\frac{d r}{d t}=u+\frac{\left\langle F_{\mathrm{DEP}}\right\rangle}{6 \pi \eta a}+\left(\rho_{p}-\rho_{m}\right) \frac{2 a^{2}}{9 \eta} g .
$$

With this simple flow model, the motion of the particles can be analyzed by using dynamical systems methods. Two different dynamical phenomena are thus unveiled. Far from the electrodes the flow is only a small perturbation of the quiescent state. Thus, the invariant line of fixed points that in absence of flow is located where the $n$-DEP force balances the positive buoyancy, disintegrates into a discrete chain of interconnected saddles and nodes. Because of normal hyperbolicity [12], the invariant manifold originally formed by a continuum of fixed points is preserved with just a slight change of shape as the saddle-node connecting manifold. This, however, induces a dramatic change in the dynamics since hyperbolic fixed points repel the particles which then accumulate in small regions near the nodes as depicted in Figs. 3(a) and 3(b). There the 
trajectories of several particles submitted to $n$-DEP forces are shown to convergence towards equilibrium points situated above the interelectrode gaps. Analogously, Figs. 3(c) and 3(d) show for $p$-DEP, that the particles which in absence of flow should accumulate at the edges of the electrodes can be forced by the flow, to concentrate in the center of the electrodes instead. Experimental evidence for the accumulation of particles in small regions above the electrodes has been reported for both $n$-DEP $[3,13]$ and $p$-DEP $[6,14]$ but without reference to the dynamical origin of the phenomenon.

A second-and stronger-dynamical effect takes place closer to the electrode surfaces: the creation of a closed zone from which particles cannot escape. Figures 3(b) and 3(d) show two qualitatively different behaviors: some particles are trapped in closed areas above the gap between electrodes, whereas others escape from the flow influence converging to fixed points determined only by the DEP force. These sets of trapped orbits resemble the Stommel retention zones $[15,16]$ studied in the context of sediments, plankton, and nutrients dynamics in the ocean in the presence of the Langmuir circulation [17]. However, in contrast with this case, since the DEP force induces a non-volumepreserving dynamics, the motion within the trapping zone is "dissipative" in the dynamical systems sense. As a consequence, the particles here converge towards foci fixed points instead of circulating around centers as in the Stommel case. A phase portrait of Eq. (3) revealing this dynamical feature is shown Fig. 4(f).

Let us note that the relative importance of the DEP force to the Stokes force is proportional to $a^{2}$. Fixing the flow parameter $u_{0}$ and studying the dynamics as $a$ varies, it appears that a Stommel-like zone exists only if $a$ is smaller than a critical value $a_{c}$. The dependence $a_{c}$ on the flow strength is shown in Fig. 4(g). At $a_{c}$ bifurcations involving

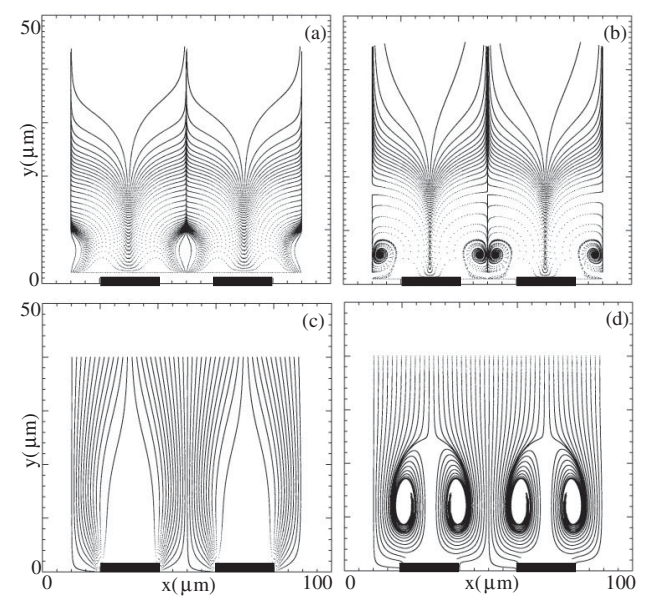

FIG. 3. (a) Particle trajectories with $n$-DEP for point II in Fig. 4 , corresponding to $\omega=5 \mathrm{MHz}, \rho_{m} / \rho_{p}=0.95, \beta=$ $0.15 d$, and $a=1.5 \mu \mathrm{m}$, with a flow moving from the gap to the electrodes. (b) For point I in Fig. 4, $a=0.75 \mu \mathrm{m}$, with the same flow as before. For the same parameters with $p$-DEP in (c) and (d), respectively. the collision and mutual annihilation of the two foci and the two saddles occur leading to the disappearance of the trapping zones. Although the detailed scenario of these bifurcations will be presented elsewhere, here we illustrate its practical impact on the particle dynamics. The righthand side panels in Fig. 3 show trapping zones for both $n$-DEP (top) and $p$-DEP (bottom) with $a_{c}<a$ whereas the left-hand side ones show no signs of the former traps for $a_{c}>a$.

We now show that dynamics can be also used to govern the behavior of the trapping zones. In analogy to the breakup of transport barriers in volume preserving steady flows [18], we found that the small time-dependent perturbations also break the trapping zones in our case. A simple way to introduce a time-dependent perturbation of the flow generated in microelectrode devices is to add a small low frequency electric field to the one used for the DEP manipulation. The electrohydrodynamic force, and therefore the resulting flow, is composed by a steady term plus an oscillatory one of twice the frequency of the applied field. At sufficiently high frequencies, the oscillatory terms are

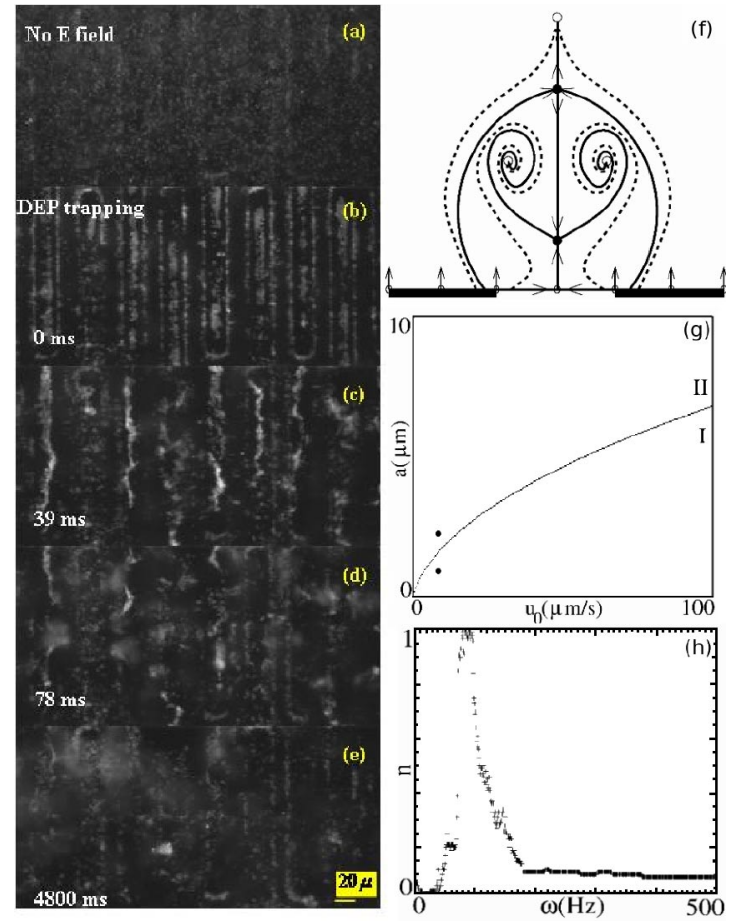

FIG. 4 (color online). (a)-(e) Image sequence showing the DEP-electro-thermoal-convective trapping of $1 \mu \mathrm{m}$ diameter latex beads and the effect of a low frequency disturbance. The potential is $10 V_{p k-p k}$, the main frequency is $10 \mathrm{KHz}$, and perturbing frequency is $100 \mathrm{~Hz}$. The focus is at 6 microns above the electrodes. (f) Phase portrait of our model, in arbitrary scales, showing the stable (white circles) and unstable (black circles) fixed points. (g) The bifurcation diagram in the parameter space $\left(a, \boldsymbol{u}_{0}\right)$, region I is where trapping occurs. (h) The ratio of particles initially within the trapping zone, escaped after 10 cycles, as a function of the frequency of perturbation, with $\epsilon=0.1$. 
comparatively small so that we can consider only the timeaveraged flow. However, if we add a small low frequency component to the applied field, it eventually will reflect as time dependence in the convective flow and the DEP force. By modeling such perturbations with a time-dependent term to the stream function,

$$
\psi=\psi_{\text {steady }}+\boldsymbol{\epsilon} \boldsymbol{u}_{0} y^{2} e^{-y / \beta} \sin (\pi x) \sin (2 \omega t),
$$

we find that the Stommel regions eventually break up allowing a complete DEP control.

In Fig. 4(h) the fraction of particles that escape from the trapping zone at a given time is plotted as a function of the frequency of the perturbation. Clearly there is value of the frequency that optimizes the particles spread, which of the order of the characteristic turnover frequency of the flow $\omega_{0} \sim \boldsymbol{u} / d \sim 10-100 \mathrm{~Hz}$. This suggests some sort of resonant driven speedup of the spreading of particles outside the trapping zone.

In order to confirm these dynamical predictions we conducted experiments on the titanium based DEP device shown in Fig. 1(b) and described in [19]. An array of $20 \mu \mathrm{m}$ titanium electrodes with a pitch of 40 microns was patterned on a titanium substrate covered with an isolation layer. A $0.2 \times 6 \mathrm{~mm}$ channel was formed by through etching a thin titanium foil 25 microns thick. By means of a syringe pump Harvard Apparatus 2000, the channel was filled with a $7.2 \times 10^{9}$ particles $/ \mathrm{mm}^{3}$ solution of fluorescent polystyrene spheres (Duke Scientific, $1.05 \mathrm{~g} / \mathrm{cm}^{3}$ density and $1 \mu \mathrm{m}$ nominal diameter) in dionized water $(2 \mu \mathrm{S} / \mathrm{cm})$ having a overall conductivity of $13 \mu \mathrm{S} / \mathrm{cm}$. Once the flow is stabilized, an ac electric field provided by a function generator Wavetek $21(11 \mathrm{MHz}$ range) is applied to the electrodes through a homemade circuit to add the perturbation. The data are collected with an epifluorescent microscope Nikon Eclipse, a $20 \times$ water immersion lens, and a CCD camera Hamamatsu C730010-12NRP.

Figure 4(a) shows stabilized flow without the influence of electrical field and the particles are uniformly suspended in the fluid. When the ac electric field $\left(10 \mathrm{KHz}, 9 V_{p k-p k}\right)$ is applied [Fig. 4(b)], the particles move toward the electrodes accumulating, as predicted, at the electrodes edges and above the center. Then a $100 \mathrm{~Hz}, 9 V_{p k-p k}$ ac signal is added and in few milliseconds [Figs. 4(c) and 4(d)] the trapping zone become unstable and the particles are dispersed in the fluid. Figure 4(e) illustrates continuous development of the perturbation.

In summary, this simple but realistic model of DEP in the presence of electrohydrodynamic convection gives us ground to predict the presence of dynamical trapping regions that are then confirmed experimentally. These are analogous to the Stommel zones found in sedimentation in convective flows, but show a different structure due to the non-Hamiltonian features of the DEP dynamics. We have shown that small time-periodic perturbations let the parti- cles escape the traps as in the Hamiltonian case. This property, that can be easily implemented in practice by superimposing a low frequency electric field, provides a simple control tool for DEP manipulations. The $p$-DEP traps described here constitute an efficient mechanism comparable to other proposed ones such as optical tweezers [20] and thermophoresis [21]. We hope that this work opens the door to more sophisticated combinations of DEP and hydrodynamic forces for control of bioparticles.

Thanks to Carl Meinhart for valuable discussions. This work is partially supported by BFM2000-1108 and REN2001-0802-C02-01 contracts. I. M. acknowledges the NSF ITR Grant No. ACI-0086061 and the NSF NIRT grant.

[1] H.A. Pohl Dielectrophoresis (Cambridge University Press, Cambridge, England, 1978).

[2] M.P. Hughes, Nanotechnology 11, 124 (2000).

[3] T. Muller, A. Gerardino, T. Schnelle, S. G. Shirley, F. Bordoni, G. De Gasperis, R. Leoni, and G. Fuhr, J. Phys. D 29, 340 (1996).

[4] N. G. Green, A. Ramos, A. Gonzalez, H. Morgan, and A. Castellanos, Phys. Rev. E 66, 026305 (2002).

[5] D. E. Chang, S. Loire, I. Mezić, J. Phys. D 36, 3073 (2003).

[6] A. Ramos, H. Morgan, N. G. Green, and A. Castellanos, J. Phys. D 31, 2338 (1998).

[7] N. G. Green, A. Ramos, A. Gonzalez, H. Morgan, and A. Castellanos, Phys. Rev. E 61, 4011 (2000).

[8] A. Castellanos, A. Ramos, A. Gonzalez, N. G. Green, and H. Morgan, J. Phys. D 36, 2584 (2003).

[9] N. G. Green, A. Ramos, A. Gonzalez, A. Castellanos, and H. Morgan, J. Electrost. 53, 71 (2001).

[10] N. G. Green and H. Morgan, J. Phys. D 31, L25 (1998).

[11] A. Ramos, A. Gonzalez, A. Castellanos, N. G. Green, and H. Morgan, Phys. Rev. E 67, 056302 (2003).

[12] S. Wiggins, Normally Hyperbolic Invariant Manifolds in Dynamical Systems (Springer-Verlag, Berlin, 1994).

[13] N. G. Green, A. Ramos, and H. Morgan, J. Phys. D 33, 632 (2000).

[14] R. Pethig, Y. Huang, X. B. Wang, and J. P. H. Burt, J. Phys. D 25, 881 (1992).

[15] H. Stommel, J. Mar. Res. 8, 24 (1949).

[16] H. Stommel, Weather 6, 72 (1951).

[17] M. A. Bees, I. Mezić, and J. McGlade, Math. Comput. Simul. 44, 527 (1998).

[18] J. M. Ottino The Kinematics of Mixing: Stretching, Chaos, and Transport (Cambridge University Press, Cambridge, England, 1989).

[19] Y. T. Zhang, E. R. Parker, M. P. Rao, M. F. Aimi, I. Mezic, and N.C. MacDonald, Proceedings of the 2004 ASME Congress, Anaheim, CA, 2004 (American Society of Mechanical Engineers, New York, 2004).

[20] S. Katsura, K. Hirano, Y. Matsuzawa, K. Yoshikawa, and A. Mizuno, Nucleic Acids Res. 26, 4943 (1998).

[21] D. Braun and A. Libchaber, Phys. Rev. Lett. 89, 188103 (2002). 